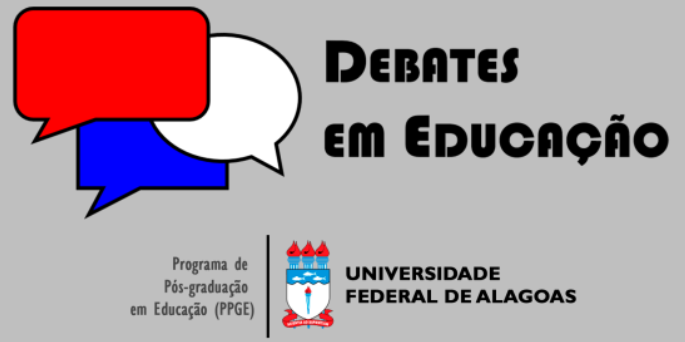

ISSN Eletrônico 2175-6600

Vol. 12 | No. 27 | Maio/Ago. | 2020

Cassiano Scott Puhl

9 iD

Pontifícia Universidade Católica do Rio Grande do Sul

(PUCRS)

c.s.puhl@hotmail.com

Thaísa Jacintho Müller

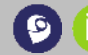

Pontifícia Universidade Católica do Rio Grande do Sul

(PUCRS)

thaisamuller@gmail.com

Isolda Gianni de Lima

(9) iD

Universidade de Caxias do Sul (UCS) iglimal@gmail.com

\section{CONTRIBUIÇÕES TEÓRICAS DA TEORIA} DE APRENDIZAGEM SIGNIFICATIVA E DO ENSINO POR MEIO DA RESOLUÇÃO DE PROBLEMAS PARA QUALIFICAR O PROCESSO DE ENSINO

\section{RESUMO}

Este artigo apresenta princípios da Teoria de Aprendizagem Significativa (TAS) e suas confluências com a Resolução de Problemas enquanto estratégia didática. $\bigcirc$ objetivo deste estudo foi identificar confluências entre a teoria e a estratégia didática, baseado principalmente nas pesquisas de Ausubel e Allevato e Onuchic, a partir da análise dos pressupostos teóricos que os caracterizam, tais como: os subsunçores, a predisposição em aprender e o material potencialmente significativo. Constataram-se aspectos similares e complementares que possuem potencial para qualificar os processos de ensino e de aprendizagem: ambas as propostas são fundamentadas no protagonismo do estudante e o entendem propulsor do desenvolvimento cognitivo e intelectual; consideram o conhecimento prévio do estudante para a construção de significados; e tem potencial para propiciar o desenvolvimento de conhecimentos, habilidades e competências para uma formação cidadã. Conclui-se, portanto, que é neles que a estratégia didática conflui com a teoria: na necessidade da predisposição em aprender, na utilização de material potencialmente significativo, nos subsunçores e na interação de conhecimentos.

Palavras-chave: Aprendizagem Significativa. Resolução de problemas. Ensino de Matemática.

\section{THEORETICAL CONTRIBUTIONS OF} SIGNIFICANT LEARNING THEORY AND TEACHING THROUGH PROBLEM SOLVING TO QUALIFY THE TEACHING PROCESS

\section{ABSTRACT}

This article presents principles of Significant Learning Theory (TSL) and its confluences with the Problem Solving as a didactic strategy. The aim of this study was to identify confluences between theory and didactic strategy, based mainly on the researches of Ausubel and Allevato and Onuchic, based on the analysis of the theoretical assumptions that characterize them, such as: the subsunitors, a predisposition to learn and potentially significant material. Similar and complementary aspects were found that have the potential to qualify learning and teaching processes: both proposals are based on the role of the student and understand him as the driver of cognitive and intellectual development; consider the student's prior knowledge for the construction of meanings; and it has the potential to foster the development of knowledge, skills and competences for citizen education. It is concluded, therefore, that it is in them that the didactic strategy converges with the theory: in the use of potentially significant material, in the subsunitors and in the interaction of knowledge.

Keywords: Meaningul Learning. Problem Solving. Mathematics Teaching.

Submetido em: 23/04/2019

Aceito em: $31 / 08 / 2019$

Publicado em: 22/06/2020

do: http://dx.doi.org/ | 0.28998/2 I 75-6600.2020v I 2n27p I 25- | 40 


\section{INTRODUÇÃO}

Os processos de ensino e de aprendizagem têm sido objeto de constante investigação, dada a dificuldade que o sistema escolar como um todo apresenta na formação de cidadãos críticos, autônomos e criativos, pelos quais clama a atual Sociedade em Rede (MORAN; MASETTO; BEHRENS, 20I3). As mudanças promovidas pelo avanço ao acesso das tecnologias - laptops, tablets, smartphones, computadores e, principalmente, a web - tornaram o conhecimento mais fácil de ser buscado por quem tenha interesse, oportunidade e aprenda a fazê-lo, modificando parte da função social da escola tradicional (MORETTO, 2007).

Diante desse contexto, o ensino meramente expositivo torna-se uma estratégia didática limitada, na qual o estudante basicamente memoriza conhecimentos que são facilmente acessíveis fora da escola, em um processo que caracteriza a aprendizagem como passiva e provisória (DEMO, 2000; PIAGET, 20 I3). No século XIX, essa estratégia didática para apresentação e registro de informações era justificável, pois o conhecimento não estava ao alcance de todos, nem mesmo recursos tecnológicos que permitissem um armazenamento seguro e o compartilhamento de conhecimentos (MORETTO, 2007), mas, no ensino contemporâneo, dar aos estudantes uma condição de espectadores mostra-se pouco eficaz e parece adequada apenas em momentos muito específicos.

O ensino meramente expositivo, geralmente, implica em uma prática pedagógica de repetição e memorização, que ainda é comum na disciplina de Matemática, em qualquer nível de escolaridade (CORDEIRO, 20I5). A estratégia didática do ensino por meio da Resolução de Problemas (RP) é uma alternativa pedagógica para reduzir a memorização sem significado de informações pelos estudantes, propiciando o seu envolvimento no processo de aprendizagem, a construção de significados a partir dos conhecimentos prévios e o desenvolvimento de habilidades e capacidades de um cidadão da Sociedade em Rede (ALLEVATO; ONUCHIC, 20I4; ALLEVATO, 20I4; CORDEIRO, 20I5; ROZAL; SOUZA; SANTOS, 2017).

A RP consiste em uma estratégia didática que possui uma organização didática que deveria prevalecer nas salas de aulas, que considera as características para formar um cidadão da Sociedade em Rede: "[...] de compartilhamento, integração, colaboração e participação integrada entre pessoas e instituições" (KENSKI, 2006, p. 95); porém, essa organização "[...] é muito distante da forma estruturada, burocraticamente hierarquizada e centralizada existente nas atuais instituições educacionais" (KENSKI, 2006, p. 95). Diante disso, os professores estão "[...] reaprendendo a conhecer, a comunicar-nos, a ensinar; reaprendendo a integrar o humano e o tecnológico; a integrar o individual, o grupal e o social" (MORAN; MASETTO; BEHRENS, 2013, p. 6I). 
Nesse contexto, elaborou-se este estudo teórico para averiguar as confluências entre os pressupostos teóricos do ensino por meio da Resolução de Problemas (RP) e da Teoria de Aprendizagem Significativa (TAS). $\bigcirc$ enfoque nesses dois princípios deve-se pela potencialidade de ambos de envolver ativamente o estudante no processo de construção de significados, possibilitando uma aprendizagem significativa, assim como no desenvolvimento de outras habilidades e capacidades, dentre elas a autonomia, a criatividade, a linguagem, a comunicação e o pensamento crítico (AUSUBEL; NOVAK; HANESIAN, 1980; AlLEVATO; ONUCHIC, 20I4; ALLEVATO, 20I4; CORDEIRO, 20I5). Sendo assim, este trabalho busca responder a seguinte questão norteadora: Quais as características confluentes do ensino por meio da resolução de problemas e da Teoria de Aprendizagem Significativa?. A seguir detalham-se os conceitos estruturantes das TAS, principalmente no que se refere à organização didática, ou seja, ao processo de ensino.

\section{TEORIA DA APRENDIZAGEM SIGNIFICATIVA}

Ausubel, Novak e Hanesian ( 1980, p. viii) afirmam que se "[...] tivesse que reduzir toda a psicologia educacional a um único princípio, diria isto: $\bigcirc$ fator isolado mais importante que influencia a aprendizagem é aquilo que o aprendiz já conhece. Descubra o que ele sabe e baseia nisso os seus ensinamentos". Os conhecimentos prévios que, na teoria da aprendizagem significativa, servem como base para a construção de novos significados, são denominados de "ideias de esteio" (AUSUBEL; NOVAK; HANESIAN, 1980) ou "subsunçores" (AUSUBEL; NOVAK; HANESIAN, 1980; AUSUBEL, 2003; MOREIRA, 2008a, 2008b, $2011)$.

Os subsunçores são, então, entendidos como a base da aprendizagem significativa. É quando o estudante possui e utiliza o que sabe para alicerçar um novo conhecimento na sua estrutura cognitiva que a aprendizagem acontece. Nesse processo de apoio ocorre a interação entre aquilo que o estudante já sabe e o novo conceito que está aprendendo, promovendo a construção de significados que atribuem mais sentido ao que era conhecimento prévio e ao novo. Como em um ciclo, os novos conceitos são agregados ou incorporados à estrutura cognitiva, transformando-a em mais específica e complexa, pronta para novas ancoragens (AUSUBEL; NOVAK; HANESIAN, 1980; AUSUBEL, 2003; MOREIRA; MASINI, 2006; MOREIRA, 1997, 2008a).

Essa interação de conhecimentos em que se fundamenta a aprendizagem significativa é mediada por uma tarefa - a estratégia didática utilizada - que busca estabelecer relações de sentido da nova informação com os subsunçores do estudante (AUSUBEL; NOVAK; HANESIAN, I 980; AUSUBEL, 2003; MOREIRA, 1997). Desse modo, ao elaborar uma tarefa de aprendizagem, faz-se pertinente considerar 
que tenham duas características: que seja não arbitrária e não literal (substantiva) no que diz respeito aos subsunçores.

A não arbitrariedade consiste, pois, da "[...] propriedade de uma tarefa de aprendizagem (por exemplo, plausibilidade, não aleatoriedade) que a torna relacionável com a estrutura cognitiva humana no sentido abstrato do termo, em alguma base 'sensata'" (AUSUBEL; NOVAK; HANESIAN, 1980, p. 525). Essa propriedade remete-se à necessidade de considerar os subsunçores do estudante, estabelecendo uma relação do conteúdo da tarefa de aprendizagem com aquilo que o estudante já sabe. $\bigcirc$ resultado dessa relação, da interação entre conhecimentos, promove as alterações na estrutura cognitiva necessárias à aprendizagem, ampliando-a (quantitativamente) e enriquecendo-a (qualitativamente). Desse modo, os conhecimentos podem ser consolidados, e não ficam como ideias soltas na estrutura cognitiva de forma arbitrária, mas vão se modificando e reconstruindo, por meio da ação mental do estudante, para a compreensão de novos conceitos (AUSUBEL; NOVAK; HANESIAN, 1980; AUSUBEL, 2003).

A substantividade, por sua vez, é uma "[...] propriedade de uma tarefa de aprendizagem que permite a substituição de elementos sinônimos sem mudança do significado ou alteração significativa no conteúdo da própria tarefa" (AUSUBEL; NOVAK; HANESIAN, 1980, p. 527). Essa definição, que até parece ser simples, é uma ideia fundamental e está relacionada a compreensão da essência de um conceito, ou seja, com a construção do significado de um conhecimento. $\bigcirc$ estudante assimila, por meio dos seus conhecimentos anteriores, um novo, e não simplesmente o repete ou o compartilha da maneira como foi recebido (AUSUBEL; NOVAK; HANESIAN, I980; MOREIRA, 1997; AUSUBEL, 2003). Desse modo, a aprendizagem não consiste apenas da memorização de palavras e de números (literal), mas implica a compreensão e a construção de significados (não literais) pelo estudante (AUSUBEL, 2003; MOREIRA; MASINI, 2006; MOREIRA, 1997, 20I Ib).

A substantividade e a não arbitrariedade são propriedades distintas, mas fortemente relacionadas, pois a compreensão do conhecimento - a construção do significado - ocorre na interação do conhecimento antigo com a informação nova, modificando ambos: o novo constrói significado e o subsunçor qualifica-se em termos de complexidade, de generalidade e de especificidade (AUSUBEL; NOVAK; HANESIAN, 1980; AUSUBEL, 2003; MOREIRA, 2008a). Nesse processo de modificação, o estudante vai, então, organizando e ordenando hierarquicamente os conhecimentos na sua estrutura cognitiva (AUSUBEL; NOVAK; HANESIAN, I980; AUSUBEL, 2003).

Os subsunçores não são os únicos fatores que influenciam na aprendizagem significativa. Um novo conhecimento é aprendido mecanicamente ou significativamente, dependendo também do nível de atenção e de disposição do estudante em aprender a tarefa de aprendizagem (AUSUBEL; NOVAK; HANESIAN, 1980; AUSUBEL, 2003). Sendo assim, o nível de significação do conhecimento depende dos subsunçores do estudante, bem como da sua intencionalidade em relacioná-lo com a tarefa de 
aprendizagem de forma não arbitrária e substantiva (predisposição em aprender), e do planejamento de estratégias didáticas que contemplem as características de um material potencialmente significativo' (AUSUBEL; NOVAK; HANESIAN, I980; AUSUBEL, 2003; MOREIRA, 2008a).

A predisposição em aprender envolve o esforço cognitivo e a disposição mental do estudante em “[...] relacionar uma tarefa de aprendizagem não arbitrariamente e substantivamente a aspectos relevantes de sua estrutura cognitiva" (AUSUBEL; NOVAK; HANESIAN, 1980, p. 523), ou seja, ela é um esforço cognitivo para estabelecer relações e compreender os conhecimentos, guardando-os, ordenando-os e organizando-os na estrutura cognitiva. Em outras palavras, o estudante precisa ter motivação e a intenção de aprender, mobilizando-se mentalmente e aumentando seu nível de atenção. De acordo com Ausubel (2003, p. 196), é, pois, "necessário um determinado grau mínimo de atenção para que ocorra a aprendizagem significativa, seguida de intenção explícita de aprendizagem e de recordação significativas".

Nesse contexto, o nível de atenção é a principal variável interveniente no processo de aprendizagem, sendo considerada como um indicativo da existência de motivação e da predisposição do estudante em aprender significativamente (AUSUBEL, 2003). Ausubel (2003, p. 202-203) entende, ainda, que "[...] a atenção é, provavelmente, uma condição cognitiva geral essencial para a ocorrência da maioria dos casos de aprendizagem significativa", uma vez que ela demonstra a intencionalidade do estudante de relacionar os conhecimentos, de forma não arbitrária e não literal, construindo novos significados. Desse modo, a predisposição não se refere somente ao querer aprender, mas consiste em "[...] uma intencionalidade, um esforço deliberado para relacionar o novo conhecimento a conhecimentos prévio, mais inclusivos, mais diferenciados, existentes na estrutura cognitiva com certa estabilidade e clareza" (MOREIRA, 2008a, p. 20).

Em relação ao material, para que seja potencialmente significativo, precisa-se igualmente contemplar os princípios da não arbitrariedade e da substantividade, organizando e planejando tarefas de aprendizagem que considerem o conteúdo didático, os subsunçores, a idade, a capacidade intelectual e mental e as condições sociais, culturais e econômicas do estudante (AUSUBEL; NOVAK; HANESIAN, 1980; AUSUBEL, 2003). O material potencialmente significativo é definido como uma "[...] tarefa de aprendizagem que pode ser aprendida significativamente, tanto porque é logicamente significativa como porque as ideias relevantes estão presentes na estrutura cognitiva particular de um aprendiz" (AUSUBEL; NOVAK; HANESIAN, 1980, p. 525).

O planejamento de um material potencialmente significativo não é tarefa simples ao professor, pois este deve atentar a certas características importantes a contemplar, a saber:

\footnotetext{
I $\bigcirc$ material didático é considerado potencial significativo, pois o significado não está no objeto em si, mas na interação de conhecimentos, ou seja, no processo cognitivo do estudante (AUSUBEL; NOVAK; HANESIAN, 1980; AUSUBEL, 2003).
} 
( I) que o próprio material de aprendizagem possa estar relacionado de forma não arbitrária (plausível, sensível e não aleatória) e não literal com qualquer estrutura cognitiva apropriada e relevante (i.e., que possui significado 'lógico') e (2) que a estrutura cognitiva particular do aprendiz contenha ideias ancoradas relevantes, com as quais se possa relacionar o novo material. A interação entre novos significados potenciais e ideias relevantes na estrutura cognitiva do aprendiz dá origem a significados verdadeiros ou psicológicos (AUSUBEL, 2003, p. I).

Há outros princípios da TAS que podem ser considerados no planejamento de um material potencialmente significativo, como da diferenciação progressiva, da reconciliação integradora, da organização sequencial e da consolidação. (AUSUBEL; NOVAK; HANESIAN, I980; MOREIRA, I997; AUSUBEL, 2003).

A diferenciação progressiva faz "[...] parte do processo da aprendizagem significativa, da retenção e da organização que resulta numa elaboração hierárquica ulterior de conceitos ou proposições na estrutura cognitiva do 'topo para baixo'” (AUSUBEL; NOVAK; HANESIAN, 1980, p. 523). Esse princípio consiste na organização dos conhecimentos e no planejamento de estratégias didáticas, de modo que os conceitos gerais e inclusivos sejam apresentados inicialmente para, posteriormente, abordar suas especificidades e os detalhes (AUSUBEL; NOVAK; HANESIAN, 1980; AUSUBEL, 2003; MOREIRA; MASINI, 2006; MOREIRA, 1997, 2008a).

A diferenciação progressiva permite que o estudante compreenda um conhecimento a partir de conceitos específicos, sendo esse um procedimento mais fácil do que compreender as especificidades de um todo. Desse modo, os conhecimentos do estudante organizam-se hierarquicamente, no qual os mais gerais e inclusivos compõem a base, o núcleo, da estrutura cognitiva e os específicos são ramificações (AUSUBEL; NOVAK; HANESIAN, 1980; NOVAK, I98I; MOREIRA, 1997; AUSUBEL, 2003).

Nesse processo ocorre a interação dos novos conhecimentos com os prévios, proporcionando a construção de significados dos conteúdos abordados na estratégia didática e reconstruindo significados, mais inclusivos, dos conhecimentos prévios (AUSUBEL; NOVAK; HANESIAN, 1980; AUSUBEL, 2003; MOREIRA; MASINI, 2006). Essa é uma consequência natural da diferenciação progressiva que origina o princípio da reconciliação integradora (AUSUBEL; NOVAK; HANESIAN, 1980; AUSUBEL, 2003; MOREIRA, 1997). Desse modo, A diferenciação progressiva e reconciliação integradora ocorrem concomitantemente, pois "à medida que aprende, o sujeito vai, progressivamente, diferenciando sua estrutura cognitiva, mas, ao mesmo tempo, tem que ir reconciliando diferenças reais ou aparentes [...]" (MOREIRA, 2008a, p. 35).

A reconciliação integradora é a "[...] parte do processo da aprendizagem significativa que resulta na delineação explícita de semelhanças e diferenças entre ideias relacionadas" (AUSUBEL; NOVAK; HANESIAN, 1980, p. 526). Esse princípio promove a compreensão de diferenças, semelhanças ou de concepções errôneas, do novo conhecimento em relação aos prévios (AUSUBEL; NOVAK; HANESIAN, 1980; AUSUBEL, 2003; MOREIRA; MASINI, 2006; MOREIRA, 1997, 2008a). 
A reconciliação integradora tem a função de qualificar os conhecimentos prévios, ao unificar os conceitos semelhantes na estrutura cognitiva, permitindo a construção de significados mais específicos e abrangentes; e reconciliar inconsistências reais ou aparentes estabelecidas pela interação entre os conhecimentos dos estudantes (AUSUBEL; NOVAK; HANESIAN, I980; AUSUBEL, 2003; MOREIRA; MASINI, 2006; MOREIRA, 2008a).

Outro princípio da TAS é a organização sequencial. Esse princípio organiza os conteúdos, buscando propiciar a construção de significados pela "[...] diferenciação progressiva dos conceitos ou proposições com o consequente refinamento dos significados e um aumento potencial para a criação de uma base para posterior aprendizagem significativa" (AUSUBEL; NOVAK; HANESIAN, 1980, p. 106).

A organização sequencial potencializa o ensino, pois os conceitos expostos inicialmente, se compreendidos e consolidados na estrutura cognitiva, tornam-se conhecimentos prévios para a construção de novos significados (MOREIRA, 1997; AUSUBEL, 2003). Nesse processo proporciona-se um avanço gradativo em nível de especificidade e de compreensão, permitindo "[...] que cada progresso alcançado na aprendizagem sirva como uma base apropriada e uma função de ancoragem para a aprendizagem e a retenção de itens subsequentes na sequência ordenada" (AUSUBEL, 2003, p. 17I).

Associado a organização sequencial tem-se o princípio da consolidação, que consiste da "[...] confirmação, correção e clarificação, no decurso do retorno (feedback), e através da prática diferencial e da revisão, no decurso da exposição repetida, com retorno, ao material de aprendizagem" (AUSUBEL, 2003, p. 172). A consolidação proporciona “[...] uma prontidão contínua de matérias e um êxito na aprendizagem sequencialmente organizada" (AUSUBEL, 2003, p. 172). Diante disso, faz-se pertinente verificar, continuamente, o nível de compreensão dos conceitos estudados e das ressignificações dos conhecimentos prévios, pois a existência de uma estrutura cognitiva estável e concisa torna possível o estabelecimento de novas interações e a construção de novos significados, em um nível maior de complexidade (AUSUBEL; NOVAK; HANESIAN, 1980; AUSUBEL, 2003).

\section{A RESOLUÇÃO DE PROBLEMAS PARA ENSINAR E APRENDER MATEMÁTICA}

A Resolução de Problemas (RP) consiste em uma estratégia de ensino utilizada principalmente na área da Matemática, que passou a ter ênfase em estudos e pesquisas na década de 1930, desenvolvendose sob os fundamentos do construtivismo e da psicologia cognitiva, com vistas à aprendizagem ativa e significativa (ALLEVATO; ONUCHIC, 20II; MORAIS; ONUCHIC, 20I4). Essa estratégia objetiva qualificar os processos de ensino e aprendizagem (POLYA, 1985; FREITAS, 2008; VAN DE WALLE, 2009), promovendo a contextualização do conteúdo por meio de um ensino globalizado. 
A RP desenvolveu-se durante décadas, adaptando-se às mudanças da sociedade, especialmente da realidade educacional, e ao desenvolvimento tecnológico (ALLEVATO; ONUCHIC, 20II; MORAIS; ONUCHIC, 20I4). Existem, ao menos, três maneiras distintivas de abordar e conceber a RP, que são: ensinar sobre resolução de problemas, ensinar para resolução de problemas e ensinar por meio da resolução de problemas, sendo que o conteúdo, como objetivo didático, é a principal característica que as diferem (SCHROEDER; LESTER, 1989 apud ONUCHIC; ALLEVATO, 2004, 20 II; ALLEVATO, 20I4; MORAIS; ONUCHIC, 20I4).

Neste trabalho, utiliza-se a abordagem do ensino por meio da RP, por se tratar de uma estratégia didática que está em consonância com os referenciais dos Parâmetros Curriculares Nacionais (PCN), das Orientações Curriculares Nacionais para o Ensino Médio (PCNEM) e da Base Nacional Comum Curricular (BNCC), nas quais há o entendimento de que o ensino dos conteúdos matemáticos potencializa-se quando se inicia por um problema (BRASIL, 1999, 2002, 2006a, 2006b, 2018). Assim, "[...] o problema é visto como ponto de partida para a construção de novos conceitos e novos conteúdos; os alunos sendo coconstrutores de seu próprio conhecimento e, os professores, os responsáveis por conduzir esse processo" (ONUCHIC; ALLEVATO, 201 I, p. 80).

A função do problema², não é somente dar origem aos processos de ensino e de aprendizagem, mas de desafiar, ativar esquemas mentais do estudante para resolver a situação e tomar decisões. Nessa perspectiva, o problema caracteriza-se como uma situação que o estudante não tem conhecimento, ou algoritmos prescritos, para resolvê-lo de forma imediata, no qual demonstra interesse em compreender e resolvê-lo (VAN DE WALLE, 2009; ALLEVATO; ONUCHIC, 20। I; GONÇALVES, 20I 5). Além disso, didaticamente, o problema possui a característica fundamental de servir como uma ponte cognitiva entre o que o estudante sabe e o que precisa saber (POZO; ANGÓN, 1998).

O problema tende a desafiar e a instigar o estudante a pensar, a realizar um esforço cognitivo para compreender a problemática e para resolvê-lo (ALLEVATO; ONUCHIC, 20II; BROLEZZI, 20I3; CORDEIRO, 20I5). Desse modo, caracteriza-se como uma situação de aprendizagem, pois o estudante não tem conhecimentos suficientes para resolvê-lo e precisa se envolver no planejamento estratégias de resolução, compartilhamento de conhecimentos - com colegas ou com o professor -, verificação hipóteses e análise de resultados. $\bigcirc$ ensino por meio da RP possibilita a realização de um trabalho cooperativo, no qual o estudante não somente está construindo significados, mas também desenvolve habilidades (AUSUBEL; NOVAK; HANESIAN, 1980; ECHEVERRÍA; POZO, I998; DINIZ, 2007; ALLEVATO; ONUCHIC, 20I4; ALLEVATO, 20I4; PIEKARSKI; RIBAS, 20I6).

\footnotetext{
${ }^{2}$ Um enunciado torna-se um problema quando o estudante precisa se questionar e refletir sobre as possíveis estratégias e sobre os conhecimentos envolvidos na resolução do problema (ECHEVERRÍA; POZO, 1998; ONUCHIC; ALLEVATO, 20I I).
} 
A compreensão dos conhecimentos envolvidos na resolução do problema promove, enfim, as mudanças na estrutura cognitiva necessárias à aprendizagem significativa, pois "[...] a solução de um dado problema envolve a reorganização dos resíduos da experiência passada para se adaptar às exigências particulares da situação problemática atual" (AUSUBEL; NOVAK; HANESIAN, 1980, p. 476). conhecimento prévio, ou subsunçores, é um fator a ser considerado no planejamento de problemas aos estudantes, influenciando o processo da resolução e a compreensão dos conhecimentos envolvidos, pois "[...] a eficiência na solução de problemas depende muito da disponibilidade e da ativação de conhecimentos conceituais adequados" (ECHEVERRÍA; POZO, 1998, p. 32). Desse modo, a TAS e a RP possuem características em comum, como o reconhecimento da influência dos subsunçores na compreensão de novos conhecimentos, entre outros que são abordadas na próxima seção.

\section{CONFLUÊNCIAS E DESAFIOS DA INTEGRAÇÃO DOS PRESSUPOSTOS TEÓRICOS}

A RP é abordada na TAS como uma estratégia didática para compor um material potencialmente significativo; isso acontece porque se considera que a estratégia didática possui potencial para proporcionar a reorganização e a modificação dos conhecimentos de estudantes por meio da interação dos subsunçores com os conhecimentos envolvidos no problema. Assim, ambos os pressupostos, a TAS e a RP, buscam desenvolver o protagonismo no estudante, envolvendo-o na realização das tarefas de aprendizagem, utilizando subsunçores para construir significados, ampliando-os quantitativamente (conhecimentos novos) e qualitativamente (conhecimento prévio) na sua estrutura cognitiva (AUSUBEL; NOVAK; HANESIAN, 1980; VAN DE WALLE, 2009; ALLEVATO, 20।4; ALLEVATO; ONUCHIC, 20I4; CORDEIRO, 20I5).

O professor possui papel fundamental no processo de ensino, ao planejar a tarefa de aprendizagem, elaborando ou selecionando problemas adequados ao contexto e ao nível cognitivo dos estudantes, abordando os conteúdos em um nível gradativo de dificuldade, "[...] num contexto de aprendizagem sequencial - em situações nas quais a capacidade de aprender um novo material pressupõe a disponibilidade do material antigo" (AUSUBEL; NOVAK; HANESIAN, 1980, p. 5I0). Corroborando com essa ideia, Gonçalves (20।5) afirma que a escolha adequada do problema, pelo professor ou pelos estudantes, é fundamental para o processo de ensino, pois, a partir dos conceitos envolvidos no problema e na sua resolução desencadeia-se o processo de compreensão e de construção de significados.

A elaboração de um problema não é procedimento simples, sendo considerado um desafio para o professor, pois na sala de aula, em qualquer nível de escolaridade, há estudantes com interesses, experiências, saberes e conhecimentos variados. $\bigcirc$ professor precisa considerar esses fatores ao elaborar uma situação que se caracterize como um desafio aos estudantes, buscando motivá-los a compreender os 
conhecimentos e a resolver o problema (POZO; ANGÓN, 1998; ALLEVATO; ONUCHIC, 20I4; ALLEVATO, 20I4; ROZAL; SOUZA; SANTOS, 2017).

Além disso, o problema precisa cumprir a sua função pedagógica: de ponte cognitiva entre o que o estudante sabe e o que precisa saber. Sendo assim, o professor precisa elaborar situações que envolvam conhecimentos não triviais e nem mesmo impossíveis de ser compreendidos, possibilitando que os estudantes utilizem conhecimentos prévios para promoverem a interação com as novas informações expostas no problema (ALLEVATO; ONUCHIC, 20I4). Essas circunstâncias dificultam a elaboração de uma situação que se efetive como um problema para uma turma, pois o estudante pode compreendê-lo como um exercício ou um problema, isso depende do conhecimento e do interesse do estudante sobre os conhecimentos envolvidos no problema (POZO; ANGÓN, 1998).

Ao elaborar problema, o professor pode considerar os princípios facilitadores da TAS (diferenciação progressiva, reconciliação integradora, organização sequencial e consolidação), disponibilizando problemas que apresentam "[...] ao aprendiz uma passagem de aprendizagem nova e sequencialmente dependente, que não pode, de forma alguma, ser dominada se não houver uma compreensão genuína da tarefa de aprendizagem anterior" (AUSUBEL, 2003, p. 130). Na solução de um novo problema, o estudante utiliza os subsunçores - conhecimentos prévios e significados construídos de um problema antecedente - para solucionar e compreender o problema em questão, mostrando indícios de ter realizado uma aprendizagem significativa.

Essa perspectiva é compartilhada Costa e Moreira (200I), ao considerar que a resolução de qualquer problema proporciona a reorganização da estrutura cognitiva, pois ocorre a interação entre subsunçores e novos conhecimentos expostos no problema. Costa e Moreira (200I, p. 264) compreendem que "se a estrutura cognitiva já possui as subsunções adequadas para permitir a reorganização do conhecimento, a resolução do problema terá cumprido o seu papel para a aprendizagem significativa". Entretanto, compreende-se que a RP não se restringe a proporcionar a aprendizagem significativa de novos conhecimentos, mas também a desenvolver habilidades e capacidades para formar cidadãos da atual sociedade.

Ao resolver o problema, o estudante precisa interpretar e compreender a problemática, elaborar e testar estratégias para solucioná-lo, uma vez que não tem conhecimento suficiente, ou um algoritmo, para resolvê-lo de forma imediata (CARVALHO, 2005; ALLEVATO; ONUCHIC, 20I4; ALLEVATO, 2014). Durante a resolução, o professor precisa incentivar o compartilhamento de ideias e de conhecimentos entre os estudantes, além de auxiliá-los na elaboração das estratégias de resolução e na compreensão dos conhecimentos envolvidos no problema (ALLEVATO; ONUCHIC, 20 I4; CORDEIRO, 2015). 
O professor, ao auxiliar o estudante, não tem uma tarefa simples, pois sua função não é de transmitir informações, mas de indicar caminhos por meio de questionamentos, sem fornecer respostas prontas (ALLEVATO; ONUCHIC, 20I4). Os questionamentos que o professor elabora tem o objetivo de fornecer subsídios para que os estudantes construam estratégias de resolução e que estabeleçam relações das novas informações com os conhecimentos prévios, possibilitando a construção de significados (ALLEVATO; ONUCHIC, 20I4).

O estudante, ao elaborar suas próprias estratégias para resolver o problema, está desenvolvendo uma atitude de investigação científica, pois precisa questionar as soluções e realizar um processo metacognitivo dos conhecimentos envolvidos no problema (SCHASTAI; SILVA; ALMEIDA, 20I2). A RP contempla o desenvolvimento de uma atitude investigativa, visto que o estudante terá que: pensar sobre a situação, elaborar estratégias, fazer registros, discutir entre pares, corrigir e validar a solução do problema (ALLEVATO; ONUCHIC, 20।4; ROZAL; SOUZA; SANTOS, 2017).

Essas ações tornam o estudante protagonista no processo de aprendizagem, no qual estabelece relações entre conhecimentos e define "[...] estratégias de solução de problemas que transcendem a simples aplicação dos princípios a exemplos autoevidentes" (AUSUBEL; NOVAK; HANESIAN, 1980, p. 526). Aceitando o desafio de resolver o problema, o estudante compartilhará ideias com colegas ou professores para compreender o problema, e, possivelmente, enfrentará questionamentos e argumentará, compartilhando conhecimentos e participando de um trabalho colaborativo na busca de uma solução. Essas ações são indicativos de um estudante ativo, motivado e predisposto a aprender (MAURI, 1999).

Desse modo, uma aprendizagem significativa com a RP requer do estudante o "[...] poder de raciocínio, perseverança, flexibilidade, ousadia, improviso, sensibilidade aos problemas e astúcia táctica além da compreensão dos princípios subjacentes" (AUSUBEL, 2003, p. I30). A RP não propicia somente a construção de significados de conhecimentos envolvidos no problema, mas tem potencial para desenvolver o estudante de forma intelectual e social (AUSUBEL; NOVAK; HANESIAN, I980; ECHEVERRÍA; POZO, 1998; ALLEVATO; ONUCHIC, 20।4; ALLEVATO, 20।4; CORDEIRO, 20। 5).

Entretanto, os estudantes não estão acostumados a resolver problemas e nem a serem protagonistas no processo de aprendizagem, sendo assim apresentam dificuldades na execução dessa estratégia didática (ALLEVATO; ONUCHIC, 20I4; ALLEVATO, 20।4; ROZAL; SOUZA; SANTOS, 20 17). Essas dificuldades são superadas quando o professor se propõe a insistir na utilização da RP, como estratégia didática, para promover a construção de significados e a formação integral do estudante (ALLEVATO; ONUCHIC, 20I4). Nessa perspectiva, Cai e Lester (2012, p. I56 apud ALLEVATO; ONUCHIC, 2014, p. 4I) advertem que 
desenvolver uma cultura de resolução de problemas em sala de aula para fazer da resolução de problemas uma parte regular e consistente de sua prática de sala de aula.

O esforço do professor em integrar a RP com os princípios da TAS é justificável, pois ambos os pressupostos teóricos não se restringem a proporcionar somente o desenvolvimento cognitivo, mas também o de outras habilidades, como a autonomia, a criatividade, a linguagem, a comunicação, as habilidades de pensamento crítico e de análise (AUSUBEL; NOVAK; HANESIAN, 1980; ECHEVERRÍA; POZO, 1998; ALLEVATO; ONUCHIC, 20I4; ALLEVATO, 20।4; PIEKARSKI; RIBAS, 20 I6). Além disso, a RP integrado com a TAS tende a reduzir a memorização de técnicas e algoritmos, para propiciar a construção de significados de conceitos e operações (CORDEIRO, 20।5). Cordeiro (20। 5, p. 99) relata que a RP "[...] abre caminhos para outras formas de aprender Matemática, mais importantes para a formação do aluno e mais significativas no contexto da sala de aula e na vida cotidiana".

Corroborando com essa ideia, Allevato (2014) afirma que nas salas de aula em que o ensino por meio da RP é adotado, é notável a satisfação dos estudantes em compreender os conceitos envolvidos. Os professores e estudantes, depois de experiências com a RP, dificilmente optam por voltar a trabalhar com uma estratégia de cunho tradicional (ALLEVATO, 20।4), pois reconhecem o avanço na formação de estudantes críticos, criativos e autônomos, que desenvolvem uma aprendizagem ativa e significativa. Desse modo, a RP integrada a TAS busca "[...] estimular a curiosidade intelectual e iniciativa, em desenvolver um pensamento independente, original e crítico, e em estimular o desejo e capacidade dos alunos para aprenderem por si mesmos" (AUSUBEL; NOVAK; HANESIAN, 1980, p. 243).

Portanto, a partir dessa análise é possível apresentar seis argumentos que são compatíveis entre a TAS e a RP: (I) o problema desafia e promove a ação cognitiva do estudante sobre os conceitos matemáticos - o processo de construção de significados - e sobre as estratégias para solucionar o problema (predisposição em aprender); (2) o processo de resolução do problema amplia o nível de abstração do estudante, dado que este precisa planejar diferentes estratégias de resolução para diferentes problemas, reconstruindo conhecimentos e construindo novos significados (interação de conhecimentos, de forma não arbitrária e substantiva, e a reorganização da estrutura cognitiva); (3) a solução de um problema proporciona ao estudante o sentimento de que é capaz de aprender matemática, aumentando a sua confiança e autoestima (predisposição em aprender); (4) o processo de resolução de um problema fornece informações contínuas, ao estudante e ao professor, que podem ser utilizadas para replanejar a estratégia de resolução ou para verificar o nível de compreensão dos conhecimentos envolvidos no problema (material potencialmente significativo); (5) a resolução de problemas promove o protagonismo do estudante e apresenta indícios da compreensão dos conhecimentos (a aprendizagem é mais significativa do que mecânica); e (6) a formalização dos conceitos pelo professor após a resolução do problema faz mais sentido, pois o estudante pode analisar e verificar os significados construídos durante a atividade 
(interação de conhecimentos, de forma não arbitrária e substantiva, e a reorganização da estrutura cognitiva) (ONUCHIC; ALLEVATO, 2004, 20I I).

\section{ASPECTOS PECULIARES ENTRE TAS E RP}

A integração da TAS com métodos de ensino - seja em atividades experimentais (MELO; OLIVEIRA; SOUZA, 2019), no uso de tecnologias (PUHL, 20।6; DUARTE, 20I7) ou na resolução de problemas (SANGOI; ISAIA; MARTINS, 20II; CORDEIRO, 20I5) - tem apresentado indícios de qualificar os processos de ensino e de aprendizagem, promovendo o protagonismo do estudante. Contudo, essa integração ainda é pouco conhecida pelos professores da Educação Básica (COSTA; MOREIRA, 200I; CORDEIRO, 2015). Diante disso, este artigo apresenta as percepções dos autores sobre o seguinte problema de investigação: Quais as características confluentes do ensino por meio da Resolução de Problemas e da Teoria de Aprendizagem Significativa? A partir da abordagem teórica realizada, constatou-se a existência de características da TAS no método da RP e de aspectos complementares entre propostas didáticas, nelas embasadas, que possuem potencial para qualificar os processos de ensino e de aprendizagem:

i) a predisposição em aprender, pressuposto da TAS, é observada na RP quando o estudante assume o desafio de solucionar o problema, definindo estratégias de resolução por meio dos subsunçores e buscando a compreensão de novos conhecimentos que envolvem o problema. Além disso, o sentimento de solucionar o problema entusiasma o estudante a avançar nos estudos e estabelecer novas relações com outros conhecimentos;

ii) o planejamento da tarefa de aprendizagem, neste caso, a elaboração do problema, deve contemplar as características de um material potencialmente significativo, ou seja, os conhecimentos envolvidos no problema devem estabelecer uma relação não arbitrária e substantiva com subsunçores. $\bigcirc$ professor precisa planejar esse material de modo que os novos conhecimentos envolvidos no problema sejam compreendidos a partir dos subsunçores; sendo assim, o nível cognitivo do estudante é fundamental para a elaboração um problema, ou seja, no planejamento desse material potencialmente significativo;

iii) o processo de resolução do problema permite que o estudante construa novos significados, tornando-se protagonista no processo de aprendizagem; há a interação dos subsunçores com os novos conhecimentos abordados no problema. Desse modo, a estrutura cognitiva amplia-se quantitativamente (conhecimentos novos) e qualitativamente (subsunçores).

A RP, portanto, possui características similares e complementares à TAS. A integração de tais pressupostos qualifica os processos de ensino e de aprendizagem, tornando o estudante protagonista e possibilitando uma aprendizagem significativa dos conhecimentos envolvidos. Além disso, disponibilizar 
problemas ao estudante é uma forma de propiciar o desenvolvimento da autonomia, da criatividade, da criticidade, da comunicação e da busca por conhecimentos, colaborando para a formação de um cidadão para a Sociedade em Rede, conforme está previsto na BNCC.

\section{REFERÊNCIAS}

ALLEVATO, N. S. G. Trabalhar através da resolução de problemas: possibilidades em dois diferentes contextos. VIDYA, Santa Maria, v. 34, n. I, p. 209-32, 2014.

ALLEVATO, N. S. G.; ONUCHIC, L. R. Ensino-Aprendizagem-Avaliação de Matemática: por que Através da Resolução de Problemas? In: ONUCHIC, L. R. et al. (org.). Resolução de Problemas: teoria e prática. Jundiaí: Paco Editorial, 20 | 4.

ALLEVATO, N. S. G.; ONUCHIC, L. R. Pesquisa em resolução de problemas: caminhos, avanços e novas perspectivas. Bolema. Rio Claro, n. 4I, p. 73-98, dez. 201 I.

AUSUBEL, D. P. Aquisição e retenção de conhecimento: uma perspectiva cognitiva. Lisboa: Paralelo, 2003.

AUSUBEL, D. P.; NOVAK, J. D.; HANESIAN, H. Psicologia Educacional. 2. ed. Rio de Janeiro: Interamericana, 1980.

BRASIL. Ministério da Educação. Base Nacional Comum Curricular: Educar é a base. Brasilia: MEC, 2018.

BRASIL. Orientações curriculares para o ensino médio. Brasília: MEC, 2006a.

BRASIL. Parâmetros Curriculares Nacionais: Ensino Médio. Ciências da Natureza, Matemática e suas Tecnologias Brasília: Ministério da Educação, 1999.

BRASIL. Parâmetros Curriculares Nacionais Ensino Médio (PNCEM): orientações complementares aos parâmetros curriculares nacionais. Brasília: MEC/SEMT, 2006b.

BRASIL. Secretaria de Educação Média e Tecnológica. PCN + Ensino Médio: orientações educacionais complementares aos Parâmetros Curriculares Nacionais: ciências da natureza, matemática e suas tecnologias. Brasília: Ministério da Educação, 2002.

BROLEZZI, A. C. Criatividade e resolução de problemas. São Paulo: Livraria da Física, 2013.

CARVALHO, M. Problemas? Mas que problemas?!. Estratégias de resolução de problemas em sala de aula. Petrópolis: Vozes, 2005.

CORDEIRO, E. M. Resolução de problemas e aprendizagem significativa no ensino de Matemática. 20।5. I08f. Dissertação (Mestrado). Universidade Federal de Uberlândia, Uberlândia, 2015.

COSTA, S. S. C.; MOREIRA, M. A. A resolução de problemas como um tipo especial de aprendizagem significativa. Caderno Brasileiro de Ensino de Física, Florianópolis, v. I8, n. 3, p. 263-276, jan. 200 I.

DEMO, P. Educar pela pesquisa. 4.ed. Campinas, SP: Autores Associados, 2000. 
DINIZ, M. I. Resolução de problemas e comunicação. In: SMOLE, K. S.; DINIZ, M. I. Ler, escrever e resolver problemas: habilidades básicas para aprender Matemática. Porto Alegre: Artmed, 2007.

DUARTE, D. W. A. Q-memória: um jogo digital para o estudo de química. Debates em Educação, Maceió, v. 9, n. 19, p. |55-161, dez. 2017.

ECHEVERRÍA, M. D. P. P.; POZO, J. I. Aprender a resolver problemas e resolver problemas para aprender. In: POZO, J. I. (org.). Solução de problemas: aprender a resolver, resolver para aprender. Porto Alegre: ArtMed, 1998.

FREITAS, J. L. M. Teoria das Situações Didáticas. In: MACHADO, S. D. A. (org.). Educação Matemática: Uma (nova) introdução. $3^{a}$ ed. São Paulo: EDUC, 2008.

GONÇALVES, R. Resolução de problemas: uma proposta para a aprendizagem significativa das funções definidas por várias sentenças. 2015. 26f. Produto educacional (Mestrado em Ensino de Ciências e Matemática). Universidade Cruzeiro do Sul, São Paulo, 2015.

KENSKI, V. M. Tecnologias e ensino presencial e a distância. 3. ed. Campinas, SP: Papirus, 2006.

MAURI, T. O que faz com que o aluno e a aluna aprendam os conteúdos escolares?. In: COLL, César et al. O construtivismo na sala de aula. 6. ed. São Paulo: Ática, 1999.

MELO, C. C.; OLIVEIRA, R. C. B.; SOUZA, A. N. Utilização de experimentação como aporte em atividades problematizadoras para a significação de conceitos químicos no Ensino Básico. Debates em Educação, Maceió, jul. 2019.

MORAIS, R. S.; ONUCHIC, L. R. Uma Abordagem Histórica da Resolução de Problemas. In:

ONUCHIC, L. R. et al. (Org.). Resolução de Problemas: teoria e prática. Jundiaí: Paco Editorial, 20 I 4.

MORAN, J. M.; MASETTO, M. T.; BEHRENS, M. A. Novas tecnologias e mediação pedagógica. 21 . ed. Campinas, SP: Papirus, 2013.

MOREIRA, M. A. A teoria da Aprendizagem Significativa segundo Ausubel. IN: MASINI, E. A. F. S.; MOREIRA, M. A (Org.). Aprendizagem significativa: condições para ocorrência e lacunas que levam a comprometimentos. São Paulo: Vetor, 2008a.

MOREIRA, M. A. Aprendizaje significativo: un concepto subyacente. In: ENCUENTRO INTERNACIONAL SOBRE EL APRENDIZAJE SIGNIFICATIVO, 2., 1997, Burgos. Actas... Burgos (Espanha), 1997.

MOREIRA, M. A. O que é afinal aprendizagem significativa. In: MOREIRA, M. A. Aprendizagem significativa: a teoria e textos complementares. São Paulo: Livraria de Física, 201 I .

MOREIRA, M. A. Organizadores previos y aprendizaje significativo. Revista Chilena de Educación Científica, v. 7, n. 2, p. 23-30, 2008b.

MOREIRA, M. A.; MASINI, E. A. F. S. Aprendizagem significativa: a teoria de David Ausubel. 2. ed. São Paulo: Centauro, 2006.

MORETTO, V. P. Prova: um momento privilegiado de estudo, não um acerto de contas. Rio de Janeiro: Lamparina, 2007. 
ONUCHIC, L. R.; ALLEVATO, N. S. G. Novas reflexões sobre o ensino-aprendizagem de matemática. In: BICUDO, M. A. V.; BORBA, M. C. (Org.). Educação matemática: pesquisa em movimento. São Paulo: Cortez, 2004.

ONUCHIC, L. R.; ALLEVATO, N. S. G. Pesquisa em Resolução de Problemas: caminhos, avanços e novas perspectivas. Boletim de Educação Matemática, Rio Claro, v. 25, n. 4I, p. 73-98, dez. 201 I.

PIAGET, J. A psicologia da inteligência. Petrópolis: Vozes, 2013.

PIEKARSKI, M.; RIBAS, J. L. D. Resolução de problemas em matemática: um olhar a partir da Aprendizagem Significativa de Ausubel. In: PARANÁ. Secretaria de Estado da Educação. Os desafios da escola pública paranaense na perspectiva do professor PDE, 20I3. Curitiba: SEED/PR, 2016.

POLYA, G. O ensino por meio de problemas. Revista do Professor de Matemática. São Paulo, SBM, n. 7, p. II-16, 1985.

POZO, J. I.; ANGÓN, Y. P. A solução de problemas como conteúdo procedimental da educação básica. In: POZO, J. I. et al. (Org.). A solução de problemas: aprender a resolver, resolver para aprender. Porto Alegre: Artmed, 1998.

PUHL, C. S. Números complexos: interação e aprendizagem. 20 16. 244f. Dissertação (Mestrado Profissional). Universidade de Caxias do Sul, Caxias do Sul, 2016.

ROZAL, E. F.; SOUZA, E. S. R.; SANTOS, N. T. Aprendizagem em matemática, aprendizagem significativa e neurociência na educação dialogando aproximações teóricas. Revista REAMEC, Cuiabá, v. 5, n. I, p. 143-164, jan./jun. 2017.

SANGOI, E,; ISAIA, S. M. A.; MARTINS, M. M. Aprendizagem significativa da derivada com o uso do software Maple através da metodologia da resolução de problemas. Vidya, Santa Maria, v. 31, n. I, p. 99-I I0, jan./jun., 201 I.

SCHASTAI, M. B.; SILVA, S. C. R.; ALMEIDA, M. F. M. Resolução de problemas - Uma perspectiva no ensino de matemática. Revista Brasileira de Ensino de Ciência e Tecnologia, Ponta Grossa, v. 5, n. 3, p. 52-69, jan. 2012.

VAN DE WALLE, J. A. Matemática no ensino fundamental. 6. ed. Porto Alegre: Artmed, 2009. 